\title{
Taxonomía y antecedentes biológicos de Microdulia mirabilis (Rothschild 1895) (Lepidoptera: Saturniidae)
}

\section{Taxonomy and biological antecedents of Microdulia mirabilis (Rothschild 1895) (Lepidoptera: Saturniidae)}

\author{
LUIS E. PARRA ${ }^{1 *}$ \& HÉCtOR IBARRA-VIDAL ${ }^{2}$ \\ ${ }^{1}$ Departamento de Zoología, Universidad de Concepción, Casilla 160-C, Concepción, Chile. \\ ${ }^{2}$ Exp Ediciones al Conocimiento, Casilla 2916, Concepción. \\ *E-mail: luparra@udec.cl
}

\begin{abstract}
RESUMEN
Se describe el huevo, larva, pupa e imago de Microdulia mirabilis (Rotschild), insecto nativo defoliador de Nothofagus obliqua Mirb. (Oerst.). M. mirabilis se distribuye entre los $35^{\circ}$ y $47^{\circ} \mathrm{S}$ en Chile y en Neuquén, Argentina. Se entregan aspectos del ciclo de vida relacionados con períodos de duración de sus diferentes estados de desarrollo. Se ilustran los distintos estados inmaduros, los imagos y las estructuras de las armaduras genitales.
\end{abstract}

Palabras clave: Cercophaninae, estados inmaduros, morfología, distribución, Nothofagus obliqua, Chile.

\begin{abstract}
The egg, larvae, pupae, and imago of Microdulia mirabilis (Rothschild), an insect that defoliates native Nothofagus obliqua Mirb. (Oerst.), are described. M. mirabilis distributes between $35^{\circ}$ and $47^{\circ} \mathrm{S}$ in Chile and Neuquén, Argentina. Aspects of the life cycle associated with the duration of different stages of development are given. Immature stages, the imago, and the genitalia are illustrated.
\end{abstract}

KEYwords: Cercophaninae, immature stages, morphology, distribution, Nothofagus obliqua, Chile.

\section{INTRODUCCIÓN}

La familia Saturniidae incluye en Chile 19 especies distribuidas en siete géneros (Angulo et al. 2004); de ellos, tres géneros: Cercophana Felder, Neocercophana Izquierdo y Microdulia Jordan, son endémicos de la Región Andina. Las especies de estos tres géneros, pertenecientes a la subfamilia Cercophaninae, son fácilmente reconocidas por las descripciones de los imagos de ambos sexos, generalmente basadas en aspectos de la morfología y coloración externa. Sin embargo, de sus estados inmaduros y biología se tiene sólo información parcial (Bartlett-Calvert 1894, Izquierdo 1895, Silva-Figueroa 1920, Ureta 1944, Artigas 1994).

De estas tres entidades naturales, Microdulia Jordan es un género monoespecífico; M. mirabilis Rothschild constituye el satúrnido más pequeño de Chile y es diagnosticado por la ausencia de la epífisis en las tibias anteriores de la hembra (Jordan 1924) y la ausencia de una cola en las alas posteriores del macho y de la hembra (Jordan 1924; Angulo et al. 2004). La especie se ha descrito sobre la base de caracteres externos a partir de escasos ejemplares. No existen antecedentes sobre sus estados inmaduros y biología, mientras sólo existe una breve descripción del capullo de la pupa (Jordan 1924, Silva-Figueroa 1920, Ureta 1944).

El hallazgo de algunas larvas de Microdulia mirabilis de cuya crianza emergió un imago permitió reunir nuevos antecedentes de uno de los satúrnidos más escasos en las colecciones entomológicas. A partir de estos antecedentes, el presente trabajo entrega una descripción exhaustiva del 
imago y los estados inmaduros de M. mirabilis así como de otros aspectos de su ciclo de vida.

\section{MATERIALES Y MÉTODOS}

Para el presente estudio se colectaron larvas de Microdulia mirabilis en la localidad de Pucoihue (20⒉ $\left.29^{\prime} \mathrm{S}, 7^{\circ} 21^{\prime} \mathrm{W}\right)$, $12 \mathrm{~km}$ al oeste de Osorno, Chile, las que luego fueron criadas en laboratorio hasta la obtención del adulto. Dicho adulto y otros especímenes inmaduros fueron depositados en la colección del Museo de Zoología de la Universidad de Concepción, Concepción, Chile (UCCC-MZUC) y el Museo de Historia Natural de Concepción, Chile (MHNCCL). El color de las larvas se describe a partir de las fotografías de los ejemplares. Los huevos se colectaron directamente de una postura realizada por una hembra emergida después de la crianza en laboratorio, no fueron fecundados.

Los antecedentes biológicos se obtuvieron de la literatura y de la consulta de catálogos y registros de colecta en distintos museos (Museo Nacional de Historia Natural, Santiago, Chile; UCCC-MZUC; Colección Entomológica Universidad Metropolitana de Ciencias de la Educación, Santiago, Chile). Paralelamente, se realizaron prospecciones entre Concepción y Aysén, Chile, durante diversos períodos del año.

\section{RESULTADOS}

Microdulia mirabilis (Rothschild 1895)

Cercophana mirabilis Rothschild 1895: 46

Lasicampa izquierdoi Silva 1920: 6

Microdulia mirabilis (Rothschild) Jordan 1924: 185; Ureta 1944: 62; Angulo et al. 2004: 34

\section{REDESCRIPCIÓN.}

MACHO (Fig. 1a). Cabeza castaño-anaranjada; antenas pectinadas, similar en color a la cabeza. Tórax castañoanaranjado cubierto por largas escamas pilosas. Alas anteriores: expansión alar 18-19 mm (3 machos), color castaño-anaranjado en el sector costal y área apical, con áreas amarillentas hacia el margen anal, banda antemedial castaño-anaranjada, sinuosa; banda postmedial castañoanaranjada, más gruesa hacia el margen costal que el anal; con una franja más clara entre estas dos bandas, a la altura de las venas discocelularis, sobre la cual se halla una mancha blanco-ovalada, orillada de pardo; otra faja en el tercio basal, poco marcada; franja externa clara siendo la zona apical más oscura. Alas posteriores amarillentas, atravesadas por dos franjas castaño-anaranjadas, la primera es angosta y recorre el disco al exterior de las venas discocelularis, la segunda es más ancha y toca el borde externo. Genitalia (Fig. 1b) con valvas subrectangulares con proceso del saculus espiniforme; uncus bífido, pseudouncus grueso y pequeño; aedeagus sin cornuti.

Hembra (Fig. 1c). Cabeza castaño-anaranjada, antenas simples. Tórax castaño-anaranjado cubierto por largas escamas pilosas. Alas anteriores: expansión alar 20-23 $\mathrm{mm}$ ( 5 hembras), de color pardo-rojizo, con tres franjas bien marcadas; la franja basal cenicienta, dividida en dos por la banda antemedial pardo-rojiza; entre las bandas medial y terminal con una franja cenicienta oscura, sobre la banda medial se halla una mancha oval más clara y poco conspicua; entre la banda terminal y el margen externo con una franja castaño-anaranjada, la cual es más oscura hacia la región apical. Alas posteriores con el tercio externo más oscuro que los dos tercios basales, atravesada por una franja pardo-rojiza. Genitalia (Fig. 1d) con corpus bursae y ductus bursae membranosos; corpus bursae ovalado; ductus bursae subcuadrangular; bursa copulatrix similar en tamaño al largo de las apófisis; apófisis anteriores y posteriores similares en tamaño.

DistRIBUCIÓN. Esta especie se encuentra entre las provincias de Curicó (Región de O’Higgins) y Capitán Prat (Región de Aysén) en Chile, entre los $35^{\circ}$ y $47^{\circ} \mathrm{S}$. En Argentina se registra en Aluminé, Neuquén (Dapoto et al. 2003). Su distribución conocida se corresponde con la distribución de su hospedador, un componente del bosque templado del centro sur de Chile y región Argentina adyacente.

Material examinado. CHILE. Curicó: 1 macho, El Rodal, Molina, enero 1951; Nuble: 2 hembras, Cerro Cayumanqui, 6 de febrero 2000. Arauco: 1 hembra, Peillem-Pille, Nahuelbuta, 600-800 msnm, 14 al 20 de enero 1954, LE Peña coll.; 1 hembra, Río Picoyquén, Nahuelbuta, 22 de diciembre 1962, Fetis coll. Cautín: 1 hembra, Lautaro, 10 de septiembre 1984, M. Beeche coll. Valdivia: 1 macho, Valdivia, 15 octubre 1982, C. Keim coll. Capitán Prat: comuna de Cochrane, 1 macho, 14 octubre 2006, L.E. Parra coll. (UCCC-MZUC). 1 macho, 7 agosto 1989, 1 macho enero 1980 (MHNCCL).

HuEvo (Fig. 2a-f). Ovalado; micropila con una abertura, rodeada por 10 celdas primarias espatuliformes y 16 a 17 celdas secundarias hexagonales (Fig. 2d); aeropilas dispuestas en las aristas de las celdas hexagonales en las caras laterales del huevo (Fig. 2e-f).

Material examinado. 111 huevos, Concepción 7 agosto 1998 (UCCC-MZUC). Los huevos fueron puestos por el imago en la jaula de cría.

LARVA (Figs. 3a-d y 4a-i). Cabeza: verde ceniciento, hipognatha (Fig. 4a), apéndices bucales castaño-claro. Área 
ocelar compuesta de seis ocelos, sub-iguales en tamaño, ocelos 1 y 2 muy próximos, ocelo 5 muy apegado a la base de la antena. Antena compuesta por tres antenómeros (Fig. $4 \mathrm{e})$ : primero y segundo anulares, tercero cilíndrico, tres veces más largo que los anteriores; espinerete cilíndrico, corto, posteroventral a los palpos labiales, dos veces más largo que los palpos labiales (Fig. 4c-d). Tórax: verde ceniciento en la región lateral y en la zona ventral de los scoli; verde claro e intenso en la región dorsal del scoli y tergite; con tres pares de scoli repartidos en el pro, meso y metatórax, scoli del protórax pequeño, verde claro en su extremo dorsal y con al menos cinco cerdas, scoli del meso y metatórax piramidales, con su vértices castaño claro y cerdas largas terminales. Abdomen (Fig. 3a-b): segmentos abdominales verde cenicientos en su cara lateral y verde claro e intenso en la zona dorsal; superficie dorsal del primer (A1), segundo (A2) y tercer (A3) segmento abdominal con una mancha triangular castaño claro en su margen posterior y amarillenta en su zona central anterior, el margen posterior del triángulo lleva una delgada franja amarilla que recorre todo el dorso del tercer segmento abdominal; A1 con dos manchas circulares blancas en la zona antero-lateral del dorso; A2 con dos puntos blancos, la mitad del tamaño y a la misma altura que las manchas circulares blancas de A1; scoli presente en A9 (Figs. 3a y 4i), verde ceniciento; A10 triangulariforme, verde claro intenso. Espuripedios en A3-A6 y A10, crochets biordinales, mesoseries homoídeas (Fig. 4g-h).
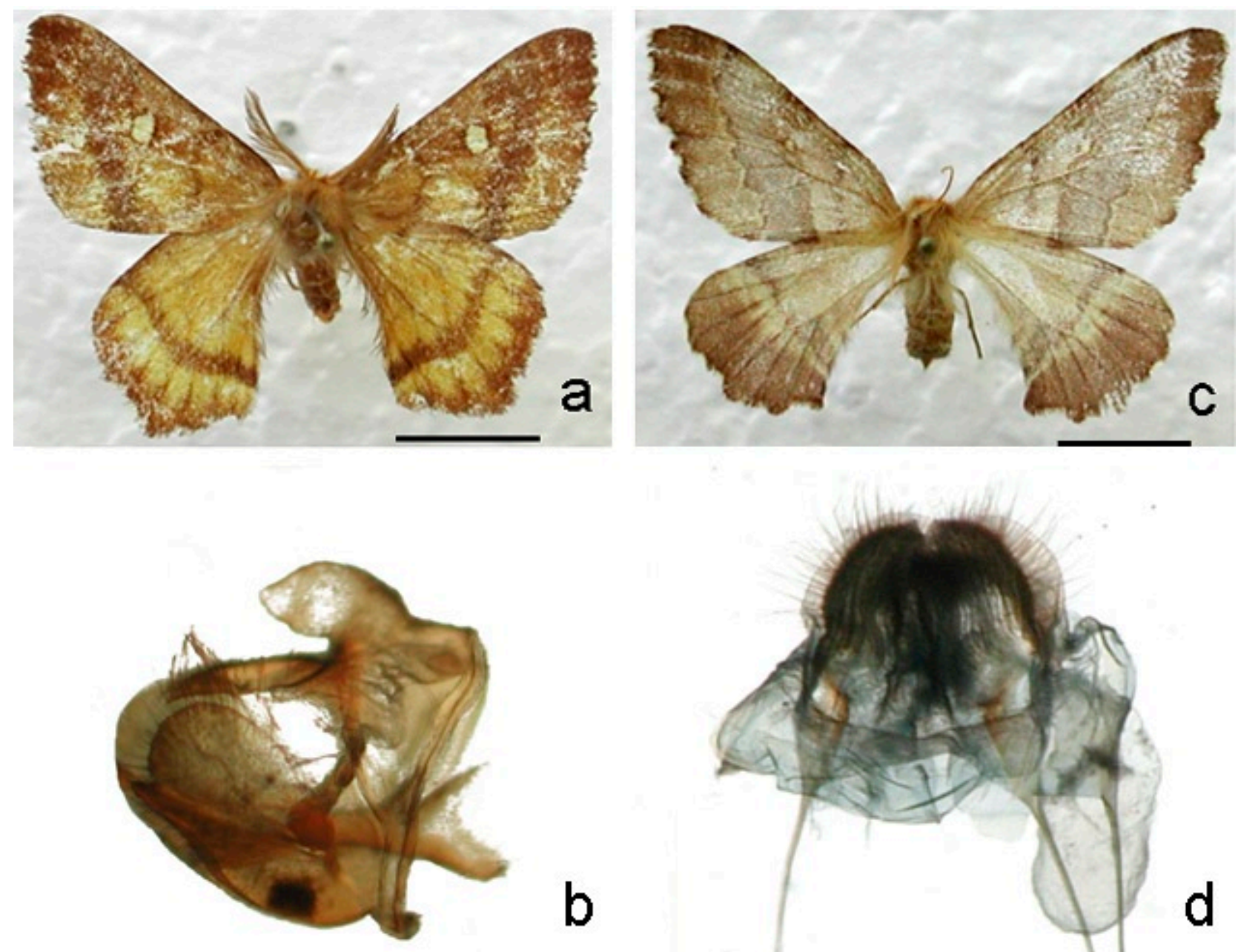

b

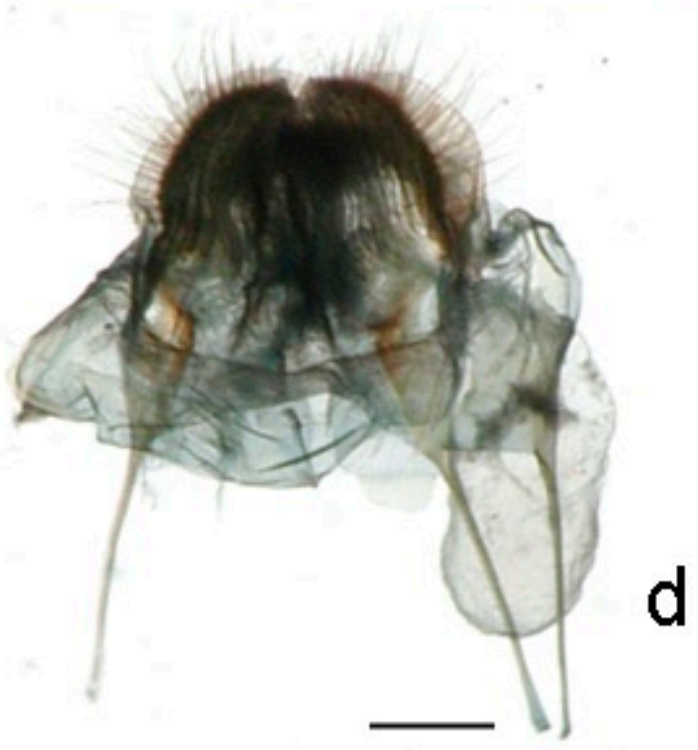

FIGURA 1. Imago de Microdulia mirabilis: a) macho, b) genitalia del macho en vista lateral; c) hembra; y d) genitalia de la hembra en vista lateral. Escala: a y b $1 \mathrm{~cm}$; c y d $1 \mathrm{~mm}$.

FIGURE 1. Imago of Microdulia mirabilis: a) male; b) male genitalia in lateral view; c) female; and d) female genitalia in lateral view. Scale: a and b $1 \mathrm{~cm}$; $\mathrm{c}$ and $\mathrm{d} 1 \mathrm{~mm}$. 

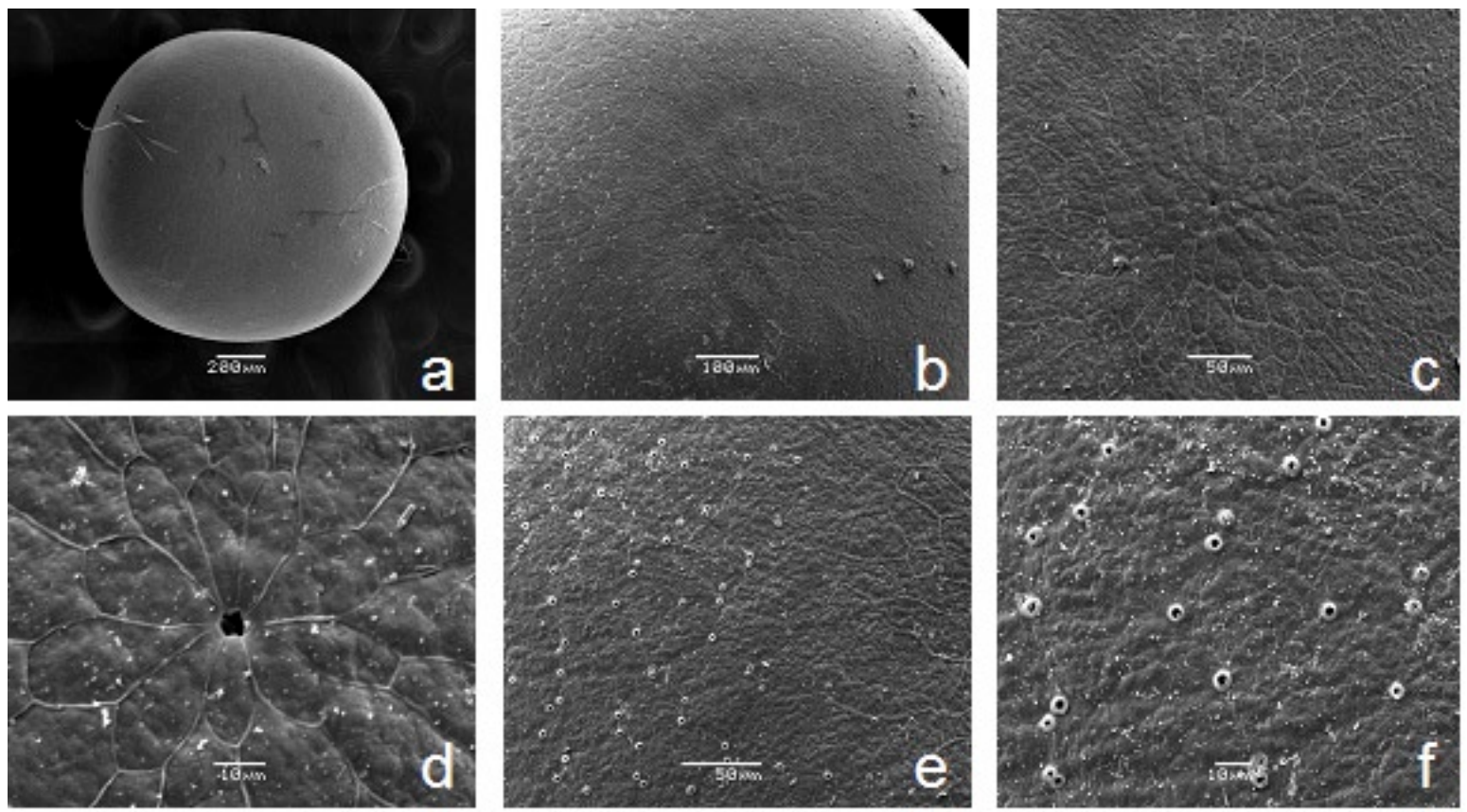

FIgURA 2. Huevo de Microdulia mirabilis: a) huevo en vista lateral; b) vista del polo anterior; c y d) área micropilar; e) escultura y aeropilas de la cara lateral; y f) aeropilas y superficie del corion.

FIGURE 2. Egg of Microdulia mirabilis: a) egg in lateral view; b) anterior pole view; c and d) micropylar area; e) aeropyles and sculpture of the lateral face; and f) aeropyles and surface chorion.

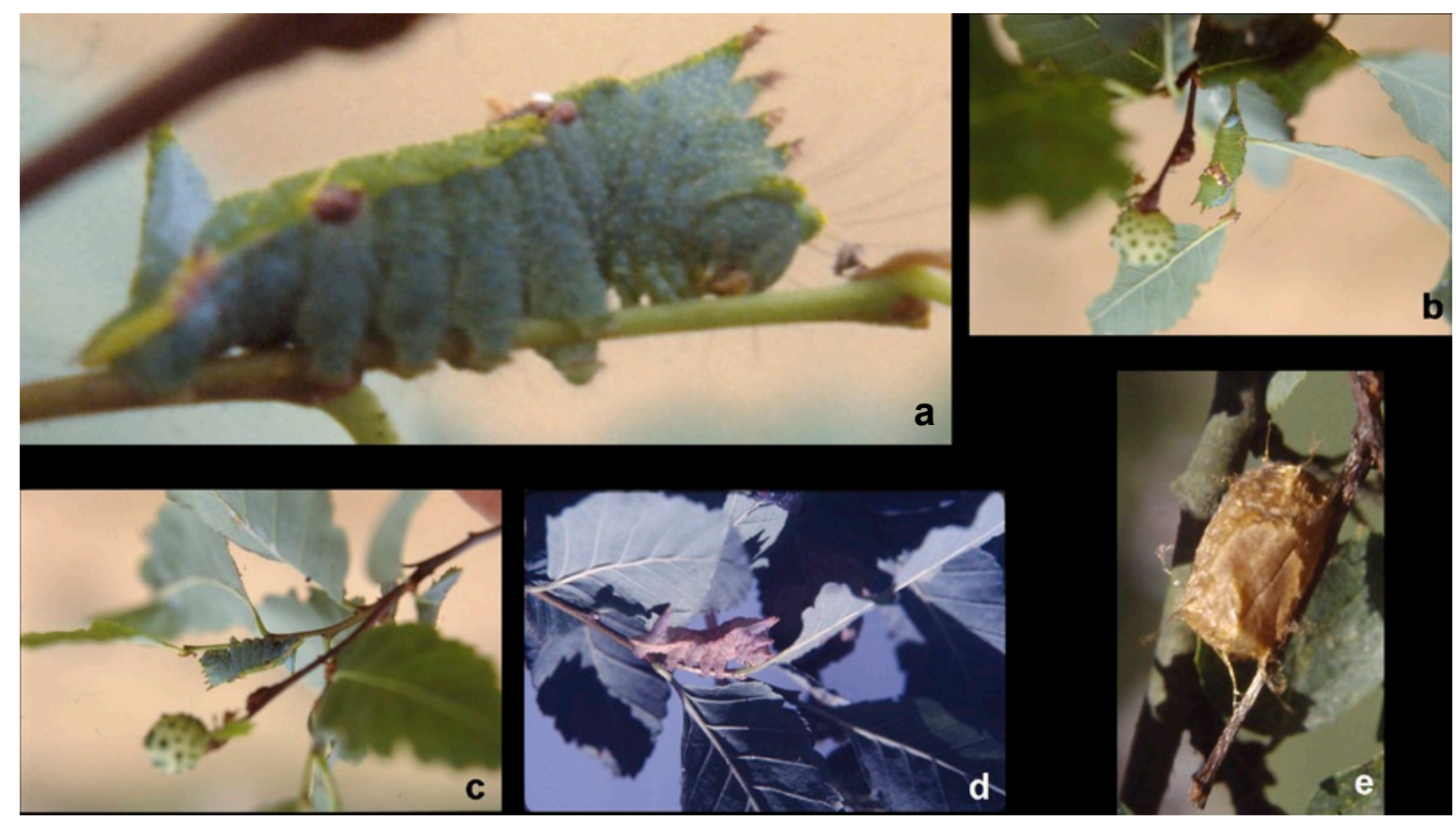

FIgURA 3. Larva y capullo de Microdulia mirabilis: a) larva en vista lateral; b y c) larvas de último estadio; d) larva en estado prepupal; y e) capullo en rama de Nothofagus obliqua.

FIgURE 3. Larvae and cocoon of Microdulia mirabilis: a) larva in lateral view; b and c) last instars larvae; d) larvae in pre-pupae stage; and e) cocoon in stem of Nothofagus obliqua. 

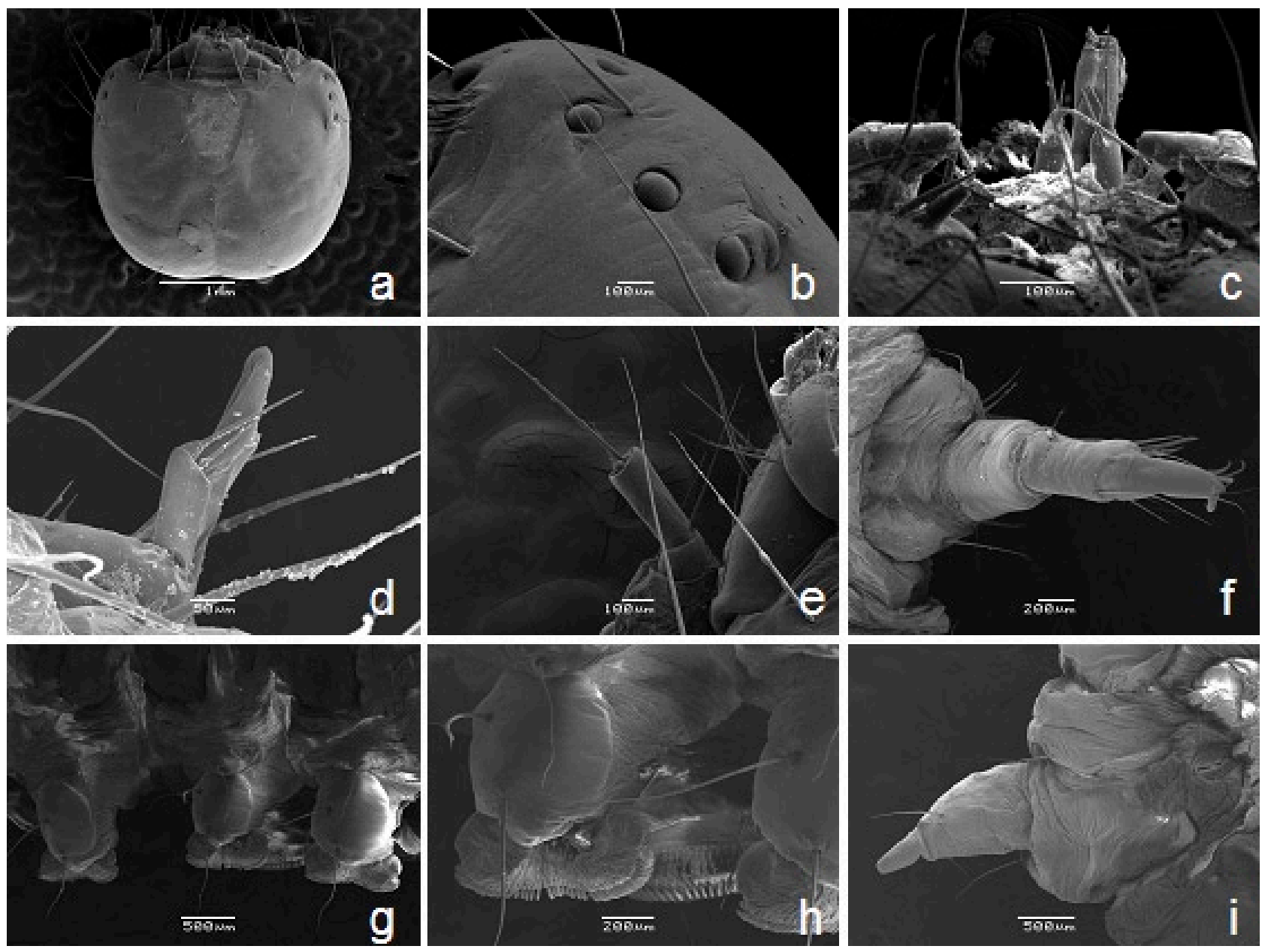

FIgURA 4. Larva de Microdulia mirabilis: a) cabeza en vista frontal; b) área ocelar; c) espinerete en vista dorsal; d) espinerete en vista lateral; e) antena; f) pata mesotorácica; g) espuripedios; h) crochets de espuripedios; e i) scoli del A9.

FIGURE 4. Larval of Microdulia mirabilis: a) head capsule in frontal view; b) stemmata area; c) spinneret in dorsal view; d) spinneret in lateral view; e) antenna; f) leg mesothorax; g) abdominal prolegs; h) crochets of abdominal prolegs; and i) dorsal scoli of the A9.

Material examinado. CHILE. Osorno: 1 larva, Pucoihue, 26 de diciembre 1990, Ibarra-Vidal coll.; 1 larva, Pucoihue, 29 de diciembre 1992, Ibarra-Vidal, coll. (UCCC-MZUC).

Capullo (Figs. 3e, 5a-b). Ovalado, blanco grisáceo a castaño-amarillento, construido en forma compacta a través del entrecruzamiento de hilos de seda. En la superficie presenta hilos de seda más gruesos que, al parecer, dan soporte a la estructura (Fig. 5b). La larva teje el capullo en torno a una rama de su hospedador (Fig. 3e).

Material examinado. CHILE. Cautín: 1 capullo, Cerro Ñielol, Temuco, 15 de noviembre 1989, Ibarra coll. (UCCC-MZUC).

Notas BiolóGICAs. El hospedero de Microdulia mirabilis es el roble (Nothofagus obliqua), de cuyos foliolos se alimentan los primeros estadios larvales junto con iniciarse el período vegetativo. Hacia mediados de primavera, la larva teje el capullo apoyándolo en un lado de una rama delgada del hospedador. El periodo pupal se extiende desde mediados de la primavera hasta fines de invierno. Una postura de huevos obtenida de un imago en laboratorio consistió en 111 huevos, los que -en condiciones de laboratorio-fueron depositados en diversos puntos de la jaula de crianza, a pesar de tener ramas de roble.

El período de vuelo, según antecedentes museológicos, de literatura (Bartlett-Calvert 1894, Rothschild 1895, SilvaFigueroa 1920, Ureta 1944, Artigas 1994, Dapoto et al. 2003, Angulo et al. 2004) y nuestras observaciones se extendería desde agosto a abril. 

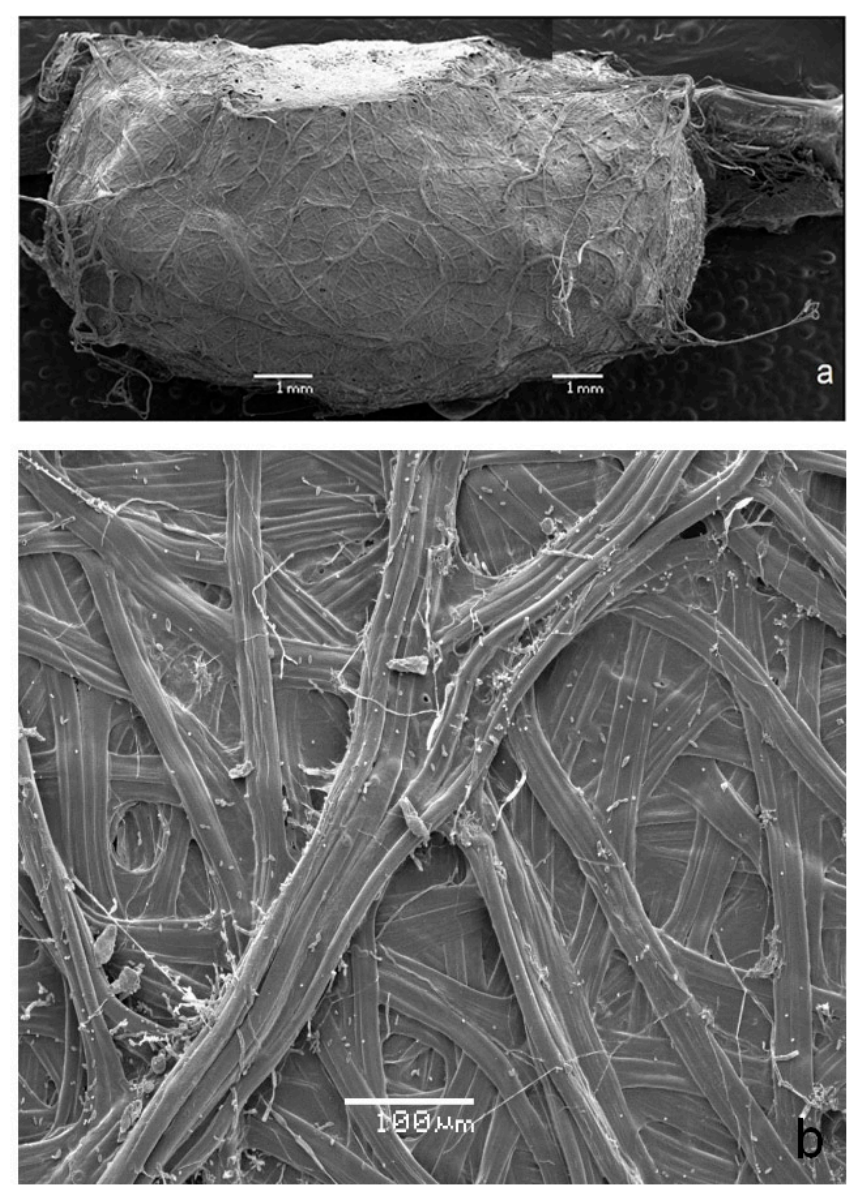

FIgURA 5. Capullo de Microdulia mirabilis: a) vista lateral; y b) detalle de la pared del capullo.

FIgURE 5. Cocoon of Microdulia mirabilis: a) lateral view; and b) details of the cocoon wall.

\section{DISCUSIÓN}

A pesar del intenso esfuerzo de recolección de larvas y pupas de Microdulia mirabilis en su hospedero (Nothofagus obliqua), se destacó la escasez de larvas encontradas, lo que se atribuiría al carácter solitario, de baja densidad y breve duración de estos estados de desarrollo. Tanto es así, que Lanfranco et al. (2001) en dos años de prospecciones y en un área de bosque de $N$. obliqua entre el Pacífico y los Andes, entre los $39^{\circ}$ y $41^{\circ} \mathrm{S}$, no recolectan la especie. Anteriormente, Carrillo y Cerda (1987), en su revisión de los zoofitófagos asociados a los Nothofagus en Chile, tampoco aluden a ella. Asimismo, la observación cuidadosa de los extremos de crecimiento reciente de las ramas del hospedador, durante el período de receso vegetativo en este árbol de hoja caduca, no arrojó una mayor capacidad de hallazgo de los capullos pupales. Si bien Lemaire \& Minet (1999) indican que en varias subfamilias de Saturniidae las larvas tienen comportamiento gregario, esta condición no aparece en M. mirabilis. Al mismo tiempo, continuamos sin conocer su lugar de oviposición, que suponemos ocurre asociado a las ramas del árbol.

Considerando que tanto la mariposa como su hospedador tienen un amplio rango de distribución, el escaso éxito del esfuerzo de recolección en distintas partes (Concepción, Osorno y Aysén) de esta gran área, como los escasos ejemplares depositados en colecciones científicas, serían un indicador de la rareza de esta especie, cuya explicación no se puede avanzar sólo con los antecedentes disponibles.

\section{AGRADECIMIENTOS}

A la Dirección de Investigación de la Universidad de Concepción por el apoyo económico a través de los proyectos 205.113.062-1.0 e Instrumental Científico 2001. Al proyecto DIUC 206.113.72-3 que financió los muestreos de LE Parra en la Región de Aysén y Exp Ediciones al Conocimiento que financió los viajes a la provincia de Osorno. A Andrés Angulo y un revisor anónimo por las sugerencias realizadas en una versión anterior de este trabajo.

\section{BIBLIOGRAFIA}

Angulo, A.O., C. Lemaire \& T.S. Olivares. 2004. Catálogo crítico e ilustrado de las especies de la familia Saturniidae en Chile (Lepidoptera: Saturniidae). Gayana 68(1):20-42.

Artigas, J.N. 1994. Entomología Económica. Insectos de interés agrícola, forestal, médico y veterinario (nativos, introducidos y susceptibles de ser introducidos). Vol. II. Ed. Universidad de Concepción, Concepción. 943 pp.

Bartlett-Calvert, W. 1894. Nuevos lepidópteros de Chile. Anales de la Universidad de Chile. Memorias Científicas 87: 133-182.

Carrillo, R. \& L. Cerda. 1987. Zoofitófagos en Nothofagus chilenos. Bosque 8(2):99-103.

Dapoto, G., H. Giganti, M. Gentili \& M. Bondoni. 2003. Lepidópteros de los bosques nativos del Departamento Aluminé (Neuquén-Argentina): II Contribución. Bosque 24:95-112.

Izquierdo, V. 1895. Notas sobre los lepidópteros de Chile. Anales de la Universidad de Chile 90:783-835.

JoRDAN, K. 1924. On the saturnoidean families Oxytenidae and Cercophanidae. Novitates Zoologicae 31:135-193.

Lanfranco, D., E. Rojas, R. Ríos \& C. Ruiz. 2001. Insect Defoliators of Nothofagus obliqua (Roble) in South Chile: Two Years Monitoring Species and Their Damage. En: Proceedings: integrated management and dynamics of forest defoliating insects (Eds. Liebhold, A.M.; McManus, M.L.; Otvos, I.S.; Fosbroke, S.L.C.). pp. 91-103.U.S. Department of Agriculture, Forest Service, Northeastern Research Station.

Lemaire, C. \& J. Minet. 1999. The Bombycoidea and their 
Gayana 74(1), 2010

Relatives. En: Lepidoptera, Moths and Butterflies. Vol. 1: Evolution, Systematics, and Biogeography (Ed. Kristensen, N.P.). pp. 321-353. Walter de Gruyter, Berlin, New York.

RothSCHILD, W. 1895. Notes on Saturniidae; with a preliminary revision of the Family down to the Genus Automeiix, and descriptions of some new species. Novitates Zoologicae 2:35-51.

Silva-FigueroA, C. 1920. Un nuevo Lasiocámpido chileno. Boletín del Museo Nacional de Historia Natural 11:5-8.

Ureta, E. 1944. La familia Saturniidae (Heterocera) en Chile. Boletín del Museo Nacional de Historia Natural 21:49-64.

Recibido: 20.10 .09

Aceptado: 08.05.10 